\title{
Changes of the liver volume and the Child-Pugh score after high dose hypofractionated radiotherapy in patients with small hepatocellular carcinoma
}

\author{
Young II Kim, MD¹, Hee Chul Park, MD¹, Do Hoon Lim, MD¹, Hyo Jung Park, MD¹, Sang Won Kang², \\ Su Yeon Park, RT${ }^{1}$, Jin Sung Kim, PhD'1, Youngyih Han, PhD'1, Seung Woon Paik, MD ${ }^{3}$ \\ 'Department of Radiation Oncology, Samsung Medical Center, Sungkyunkwan University School of Medicine, Seoul; \\ ${ }^{2}$ Department of Radiologic Science, Korea University College of Health Science, Seoul; \\ ${ }^{3}$ Department of Medicine, Samsung Medical Center, Sungkyunkwan University School of Medicine, Seoul, Korea
}

Purpose: To investigate the safety of high dose hypofractionated radiotherapy (RT) in patients with small hepatocellular carcinoma (HCC) in terms of liver volumetric changes and clinical liver function.

Materials and Methods: We retrospectively reviewed 16 patients with small HCC who were treated with high dose hypofractionated RT between 2006 and 2009. The serial changes of the liver volumetric parameter were analyzed from pre-RT and follow-up (FU) computed tomography (CT) scans. We estimated linear time trends of whole liver volume using a linear mixed model. The serial changes of the Child-Pugh (CP) scores were also analyzed in relation to the volumetric changes.

Results: Mean pre-RT volume of entire liver was 1,192.2 $\mathrm{mL}$ (range, 502.6 to 1,310.2 $\mathrm{mL}$ ) and mean clinical target volume was $14.7 \mathrm{~mL}$ (range, 1.56 to $70.07 \mathrm{~mL}$ ). Fourteen (87.5\%) patients had 4 FU CT sets and $2(12.5 \%)$ patients had 3 FU CT sets. Mean interval between FU CT acquisition was 2.5 months. After considering age, gender and the irradiated liver volume as a fixed effects, the mixed model analysis confirmed that the change in liver volume is not significant throughout the time course of FU periods. Majority of patients had a CP score change less than 2 except in 1 patient who had CP score change more than 3.

Conclusion: The high dose hypofractionated RT for small HCC is relatively safe and feasible in terms of liver volumetric changes and clinical liver function.

Keywords: Radiotherapy, Hepatocellular carcinoma

\section{Introduction}

Surgical resection, liver transplantation, and local ablation such as radiofrequency ablation (RFA) are the recommended curative treatments for small hepatocellular carcinoma (HCC) [1-3]. In clinical practice, the recommended treatments for small HCC can't always be performed. Only a small proportion of patients are candidates for curative resection because of various clinical reasons other than tumor factors [4]. Insufficient supply of the donor liver is the concern to perform transplantation [5]. RFA is the good alternative as the non-surgical modality for small HCC, however, the tumor

Received 13 September 2012, Revised 7 October 2012, Accepted 22 October 2012.

Correspondence: Hee Chul Park, MD, Department of Radiation Oncology, Samsung Medical Center, Sungkyunkwan University School of Medicine, 81 Irwon-ro, Gangnam-gu, Seoul 135-710, Korea. Tel: +82-2-3410-2605, Fax: +82-2-3410-2619, E-mail: rophc@skku.edu

(c) This is an Open Access article distributed under the terms of the Creative Commons Attribution Non-Commercial License (http://creativecommons.org/ licenses/by-nc/3.0/) which permits unrestricted non-commercial use, distribution, and reproduction in any medium, provided the original work is properly cited. www.e-roj.org 
location could be problematic causing the heat-sink effect and sometimes the serious RFA related normal tissue injuries [6].

Radiotherapy (RT) has become a palliative option in the management of HCC after the listing of RT in the HCC management guidelines by Korean Liver Cancer Study Group [1]. The listing of RT was possible through the accumulation of scientific evidences regarding the feasibility and the safety of RT used for HCC management [7-13]. Recently, there are notable suggestions that RT could be used even for small HCC in the situation which the standard treatment options are not possible to be performed $[14,15]$. Dawson [14] claimed that RT could be done with firm clinical benefit for small HCC less than $3 \mathrm{~cm}$, though she limited the use of RT for clinical trial only. Lee and Seong [15] also reported that high dose stereotactic body radiotherapy (SBRT) is indicated for small HCC when the standard options are limited by technical difficulties or patient's refusal of standard therapy.

The RT for small HCC could be done in whom the RT can be delivered safely, with an expected benefit to the patient. The authors previously reported the oncological outcomes of high dose hypofractionated RT in patients with recurrent small HCC [13]. The purpose of this study was to investigate the safety in terms of changes of liver volume and clinical liver function after high dose hypofractionated RT in patients with small HCC.

\section{Materials and Methods}

\section{Patients}

We retrospectively reviewed 20 patients with small HCC who were treated with high dose hypofractionated RT between 2006 and 2009, same with the study population of previous report by the authors [13]. The eligibility criteria for the current study were the same with the previous report. And patient with at least 2 consecutive sets of follow-up (FU) computed tomography (CT) scans were included in the study. Patient with massive intra-hepatic recurrence was excluded from the analysis. Three patients were followed with less than 3 sets of CT scans and a patient had massive intra-hepatic recurrence, thus 16 patients were enrolled in this study.

The medical record, previous RT plans, blood tests and CT scans from each patient were reviewed and analyzed retrospectively.

\section{Radiation therapy procedures}

For RT planning, contrast-enhanced CT was performed with each patient in a supine position with both arms raised above their head.

Until February 2008, CT simulation was done during free breathing using HiSpeed Advantage CT simulator (GE Medical System, Milwaukee, WI, USA). The gross tumor volume (GTV) was defined as an enhancing tumor visualized on CT images. The clinical target volume (CTV) was determined within a $1-1.5 \mathrm{~cm}$ margin of the GTV. The planning target volume (PTV) was expanded by $0.5 \mathrm{~cm}$ from the CTV. The extra margin of $1-1.5 \mathrm{~cm}$ in the craniocaudal direction was added to cover respiratory tumor motion.

Since March 2008, patients performed 4-dimentional CT simulation using the real-time position management (RPM) system (Varian Medical Systems, Palo Alto, CA, USA). Before simulation, patients practiced respiratory training aided by a housemaid biofeedback training system. Thereafter, a CT scan was obtained for the arterial and portal phase during freebreathing, and then 4-dimensional CT scans were acquired. The 4-dimensional CT simulation images were divided into 10 equal phases of breathing cycle. The 4-dimensional CT images of 0\%, 30\%, 50\%, 80\% phase and free-breathing portal phase were used to delineated targets and organs at risk volumes. The GTVs were delineated at each phase and summed up to determine the internal target volume (ITV). A $0.5-\mathrm{cm}$ margin was added to form the PTV. The extra margin of $1-1.5 \mathrm{~cm}$ in the craniocaudal direction was not added, if the 4-dimensional CT scan was used to form the PTV.

RT planning was done using a Pinnacle ${ }^{3}$ treatment planning system (TPS; ADAC Lab., Milpitas, CA, USA). High dose conformal RT was delivered as 50 Gy in daily 5 Gy fractions using 3 or more beams of 6,10 , or 15 MV X-ray.

\section{Follow-up}

After the completion of RT, patients were followed by physical examinations, blood tests (complete blood cell counts, routine chemistry, blood coagulation tests, and tumor markers) and triphasic contrast-enhanced CT scans at about 3 months interval. Pre-RT and serial 3-12 months post-RT FU CT scans were used for volumetric analysis. The serial changes of the liver volumetric parameter were analyzed. The serial changes of the Child-Pugh (CP) scores were also analyzed in relation to the volumetric changes.

\section{Liver volumetry}

The portal phase images taken at pre-RT and serial FU CT scans were restored as a fusion image sets using the Pinnacle ${ }^{3}$ TPS 
(ADAC Lab.) for each patient. One dosimetrist (KSW) contoured the serial whole liver volumes using the contouring tool in the TPS for each patient. And one radiation oncologist (PHJ) reviewed the contours whether it is appropriate. The volumes of liver region of interests (ROIs) were calculated using the internal volumetry software module installed in the TPS. And the serial volumetric measurements were extracted in the form of spread sheet using a hotscript function in the TPS.

\section{Statistics}

We estimated linear time trends of whole liver volume from pre-RT and serial FU CT scans using a linear mixed model for continuous variables (SAS PROC MIXED; SAS Institute Inc., Cary, NC, USA). A generalized linear mixed model for binary and continuous variables was used in which we adjusted for age, gender and the irradiated liver volume. In all analyses, age, gender and the irradiated liver volume were modeled as fixed effects.

Since the estimated linear time trends are based on individual changes over time, the volumetric measurements of at least two FU image sets were necessary. As a consequence, these longitudinal analyses were based on 78 sets of CT scan from 16 patients who were eligible.

All statistical analyses were performed using SPSS ver. 18.0.0 (SPSS Inc., Chicago, IL, USA).

\section{Results}

\section{Patients' characteristics}

Patient characteristics are shown in Table 1. The majority of patients were male and the median age was 62 years (range, 41 to 89 years). The Eastern Cooperative Oncology Group (ECOG) performances were less than 2 in all patients. All but 1 patient had viral etiologies to develop HCC. All patients received previous treatments including hepatic resection, RFA and transcatheter arterial chemoembolization (TACE). Treated number of tumors was more than one in 3 patients. CT simulations were done in free breathing condition for 10 patients and 4-dimensional CT simulation was done for 6 patients.

Mean pre-RT volume of entire liver was 1,192.2 $\mathrm{mL}$ (range, 502.6 to $1,310.2 \mathrm{~mL}$ ) and mean CTV volume was $14.7 \mathrm{~mL}$ (range, 1.56 to $70.07 \mathrm{~mL}$ ) (Table 1). The absolute liver volumes receiving the indicated dose $X\left(V_{X}\right)$ are listed in Table 2.
Table 1. Characteristics of patients and tumor

\begin{tabular}{|c|c|}
\hline Characteristic & No. $(\%)$ \\
\hline Age (yr), median (range) & $62(41-89)$ \\
\hline \multicolumn{2}{|l|}{ Gender } \\
\hline Male & $12(75.0)$ \\
\hline Female & $4(25.0)$ \\
\hline \multicolumn{2}{|l|}{ ECOG performance status } \\
\hline 0 & $8(50.0)$ \\
\hline 1 & $8(50.0)$ \\
\hline \multicolumn{2}{|l|}{ Etiology of liver disorder } \\
\hline Hepatitis B virus & $12(75.0)$ \\
\hline Hepatitis C virus & $3(18.8)$ \\
\hline No infection & $1(6.2)$ \\
\hline \multicolumn{2}{|l|}{ Liver cirrhosis } \\
\hline Yes & 13 (81.3) \\
\hline No & 3 (18.7) \\
\hline \multicolumn{2}{|l|}{ Child-Pugh score } \\
\hline 5 & $11(68.8)$ \\
\hline 6 & $5(31.2)$ \\
\hline \multicolumn{2}{|l|}{ Tumor numbers } \\
\hline 1 & 13 (81.3) \\
\hline 2 & $2(12.5)$ \\
\hline 3 & $1(6.2)$ \\
\hline \multicolumn{2}{|l|}{ Clinical target volume $\left(\mathrm{cm}^{3}\right)$} \\
\hline Mean & 14.7 \\
\hline$\leq 10$ & $11(68.8)$ \\
\hline $10-50$ & $3(18.7)$ \\
\hline$\geq 50$ & $2(12.5)$ \\
\hline \multicolumn{2}{|l|}{ Normal liver volume $\left(\mathrm{cm}^{3}\right)$} \\
\hline Mean & $1,192.2$ \\
\hline$\leq 999$ & $5(31.3)$ \\
\hline $1,000-1,249$ & $6(37.5)$ \\
\hline $1,250-1,499$ & $2(12.5)$ \\
\hline$\geq 1,500$ & $3(18.7)$ \\
\hline \multicolumn{2}{|l|}{ Previous therapy } \\
\hline TACE & $6(37.5)$ \\
\hline RFA & $1(6.3)$ \\
\hline $\mathrm{OP}+\mathrm{RFA}$ & $2(12.5)$ \\
\hline TACE + RFA & $7(43.7)$ \\
\hline \multicolumn{2}{|l|}{ Planning computed tomography } \\
\hline Free breathing & $10(62.5)$ \\
\hline Control breathing (4-dimensional) & $6(37.5)$ \\
\hline
\end{tabular}

ECOG, Eastern Cooperative Oncology Group; TACE, transhepatic arterial chemoembolization; RFA, radiofrequency ablation; OP, sectionectomy.

\section{Treatment outcomes}

All patients received the planned RT without interruption associated with treatment-related toxicity. There were minor anorexia and nausea in 4 and 5 patients, respectively. There was the progression of ascites after RT in 1 patient. There were 
no cases of grade 3 or greater treatment-related toxicity, such as radiation induced liver disease (RILD) or death.

The median FU after RT was 38.1 months (range, 3.9 to 75.0 months). The 3 -year overall survival was $62.5 \%$ (Fig. 1). Three patients had in-field failure and the 3-year local control was 81.3\% (Fig. 1). Intra-hepatic control rate was $31.3 \%$ at 3 years (Fig. 1). Additional TACE was performed in 11 patients.

Seven patients had in-field failure (3 patients) and intrahepatic failure (4 patients) during the serial volumetry pe-

Table 2. Clinical target volume (CTV) and irradiated normal liver volume per radiation dose

\begin{tabular}{ccc}
\hline & Mean (range) $(\mathrm{mL})$ & $\begin{array}{c}\text { Standard } \\
\text { deviation }\end{array}$ \\
\hline CTV & $14.70(1.56-70.07)$ & 20.60 \\
$V_{10}$ & $391.81(234.83-595.10)$ & 118.81 \\
$V_{20}$ & $194.39(91.64-391.03)$ & 84.85 \\
$V_{30}$ & $115.77(21.85-300.12)$ & 61.95 \\
$V_{40}$ & $65.98(7.29-144.36)$ & 33.27 \\
$V_{50}$ & $7.75(0.03-45.21)$ & 10.86 \\
\hline
\end{tabular}

A

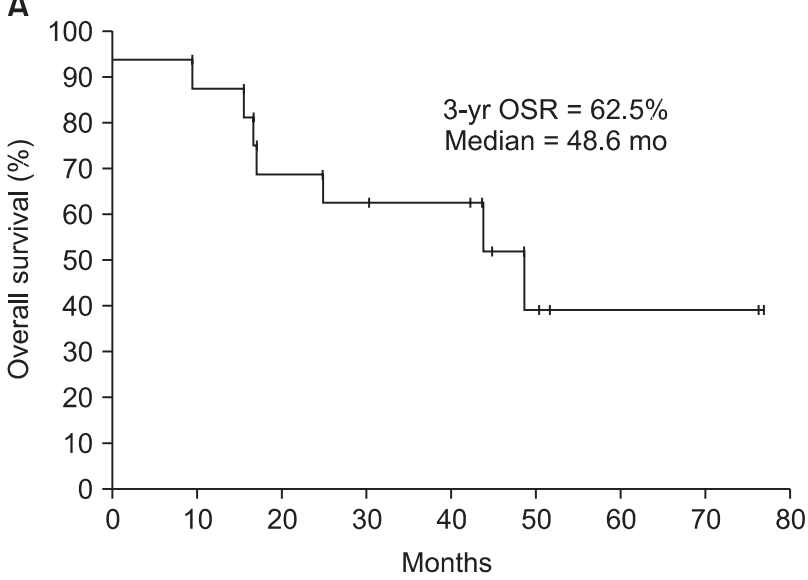

C

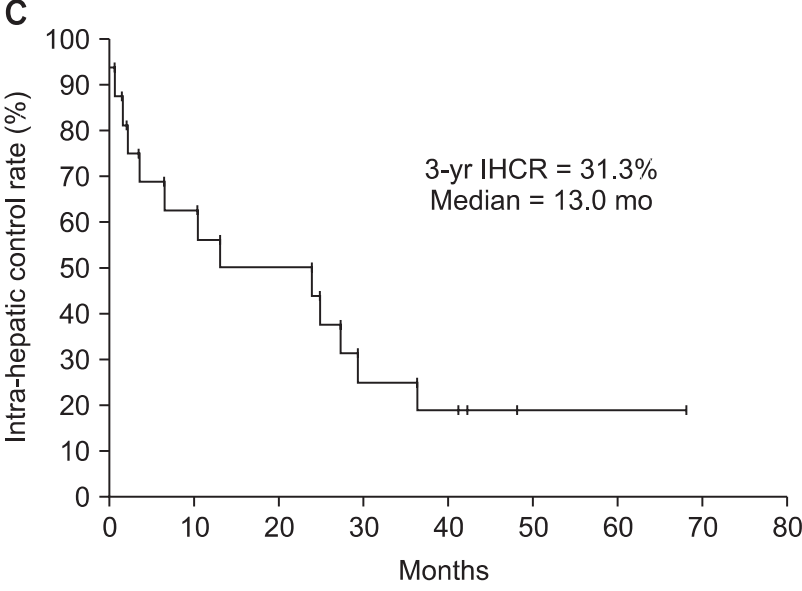

riod. Mean recurrent tumor diameter was $1.40 \mathrm{~cm}$ in infield recurrences and $1.93 \mathrm{~cm}$ in intra-hepatic elsewhere recurrences (Table 3 ).

\section{Liver volumetry}

The number of FU CT sets was different according to the patients' condition such as HCC progression or FU compliance. Fourteen (87.5\%) patients had 4 FU CT sets and 2 (12.5\%) patients had 3 FU CT sets. Mean interval between FU CT acquisition was 2.5 months.

The absolute and relative changes of liver volume measured through the FU period compared to pre-RT CT image sets are appeared (Fig. 2). After high dose hypofractionated RT for

Table 3. Size of intra-field and intra-hepatic recurrent tumors

\begin{tabular}{lccc}
\hline & $\begin{array}{c}\text { No. } \\
\text { occurred }\end{array}$ & $\begin{array}{c}\text { Mean (range) } \\
(\mathrm{cm})\end{array}$ & $\begin{array}{c}\text { Standard } \\
\text { deviation }\end{array}$ \\
\hline In-field recurrence & 3 & $1.40(0.8-2.0)$ & 0.49 \\
Infra-hepatic recurrence & 4 & $1.93(1.0-3.0)$ & 0.75 \\
\hline
\end{tabular}

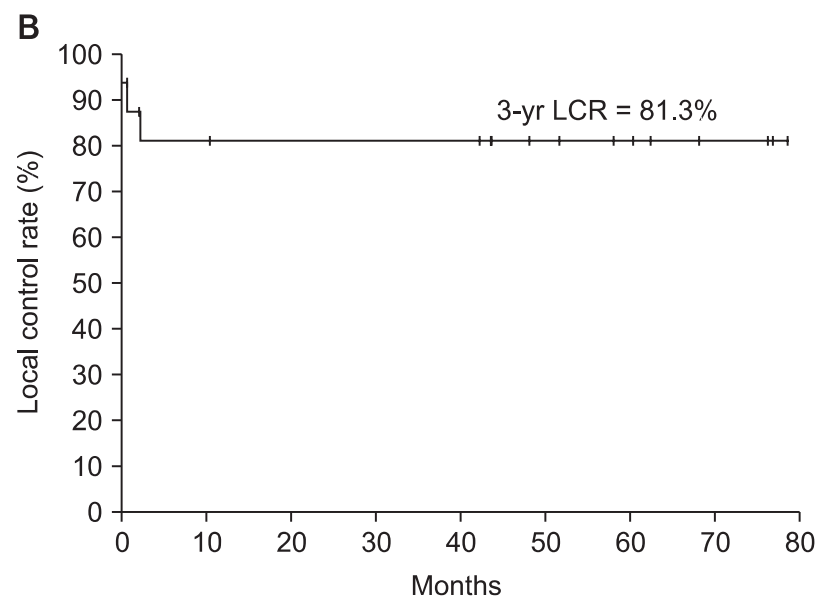

Fig. 1. Treatment outcomes; (A) the overall survival rate (OSR), (B) the local control rate $(\mathrm{LCR})$, and $(\mathrm{C})$ the intra-hepatic control rate (IHCR). 
A

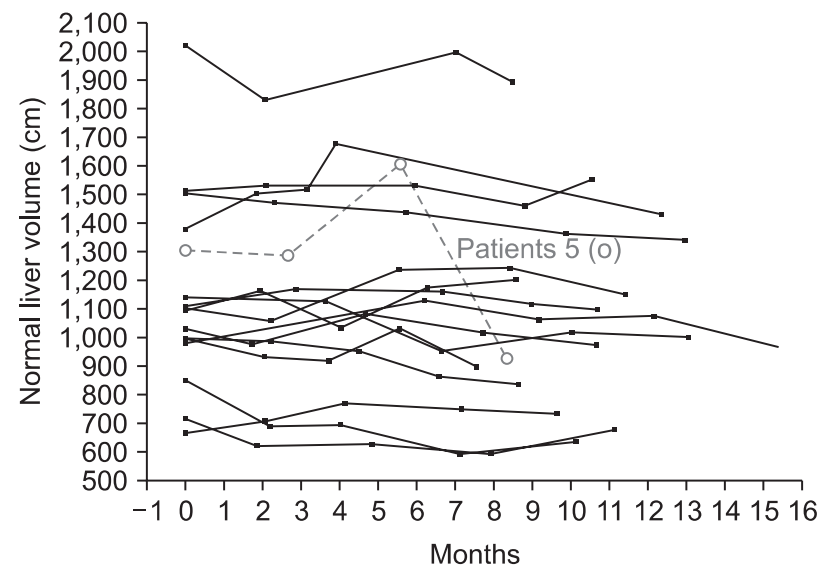

B

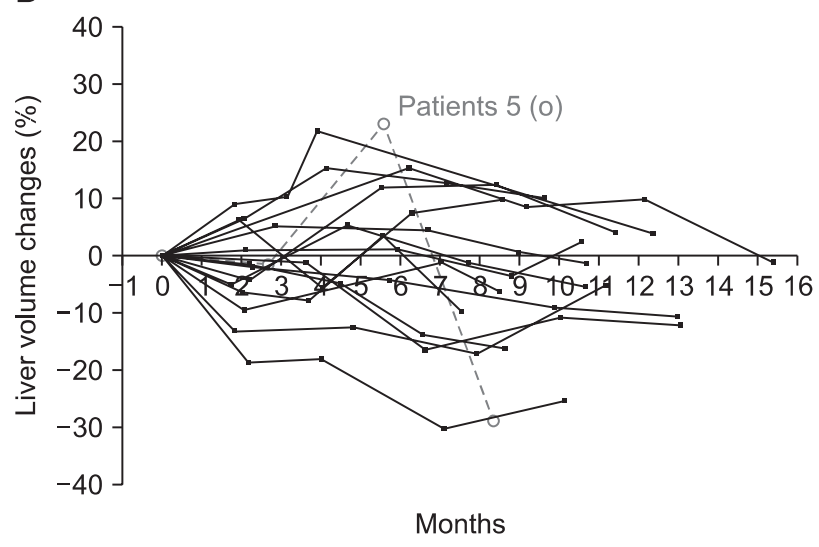

Fig. 2. The absolute (A) and relative (B) changes of whole liver volume along the follow-up periods.

Table 4. Linear mixed model analysis

\begin{tabular}{ccccc}
\hline Effect & Num DF & Den DF & F-value & $p$-value \\
\hline$V_{10} \times$ time & 4 & 68 & 0.25 & 0.9088 \\
$V_{20} \times$ time & 4 & 68 & 0.13 & 0.9719 \\
$V_{30} \times$ time & 4 & 68 & 0.11 & 0.9773 \\
$V_{40} \times$ time & 4 & 68 & 0.16 & 0.9591 \\
$V_{50} \times$ time & 4 & 68 & 0.07 & 0.9915 \\
\hline
\end{tabular}

Num DF, numerator degree of freedom; Den $D F$, denominator degree of freedom.

small HCC, there was no significant change in liver volume throughout FU period. The change in absolute volume was less than 30\% except in 1 patient (patient \#5) (Fig. 2A). After considering age, gender and the irradiated liver volume as a fixed effects, the mixed model analysis confirmed that the change in liver volume is not significant throughout the time course of FU periods (Table 4).

The relative changes were distributed between $-30 \%$ and $+30 \%$ (Fig. 2B). It seemed there are two distinct groups of patients in its relative volume changes of the liver. The compensatory increased volume followed by decreased volume was appeared in 6 patients and the initial slightly decreased volume followed by steadily preserved volume appeared in 10 patients. There was a difference in the 3 -year overall survival between two groups (66.7\% vs. $60.0 \%$ ), but it was not statistically significant $(p=0.324)$.

\section{Clinical liver function (CP score)}

The absolute $\mathrm{CP}$ score and the change of $\mathrm{CP}$ score along with the FU period are appeared (Fig. 3). The change of clinical liver function assessed in the form of CP score was not profound. Majority of patients had a CP score change less than 2 except in 1 patient who had CP score change more than 3 (Fig. 3B). This patient (patient \#5) had noticeable changes of liver volume (Fig. 2).

The patient (Patient \#5) had the progression of liver cirrhosis (LC) and the febrile cholangitis after RT. He had initial compensatory increase of liver volume about 20\% followed by the rapid shrinkage of liver volume more than $40 \%$ from its largest measurements. He died of LC progression.

\section{Discussion and Conclusion}

Many studies have reported that the lesser the volume of remnant liver, the greater the risk of liver failure in patients undergoing partial hepatectomy. Liver functional reserve is related to the quantity and quality of hepatic cells. Therefore the volume of remnant liver reflects the function of liver. In the cirrhotic condition of liver, not only the number of hepatic cell but also the extent of fibrosis influences the volume of liver. Thus, the volume of remnant liver might be overestimated to the function of liver. So, as to estimate the clinical liver function after radiation therapy for $\mathrm{HCC}$, the liver volume and the other determinants to evaluate the liver function is needed to be evaluated.

In the current study, the authors evaluated the change of liver volume and CP score as a surrogate of patients' safety after RT for small HCC. The change of liver volume and CP score was not profound in this study population. The high dose hypofractionated RT for small HCC is relatively safe and feasible to confirm the potential role as a curative modality 
A

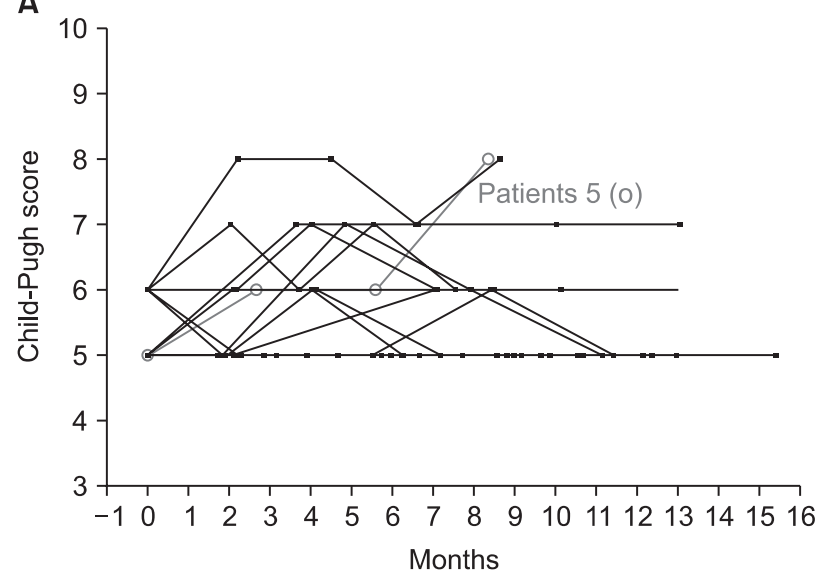

B

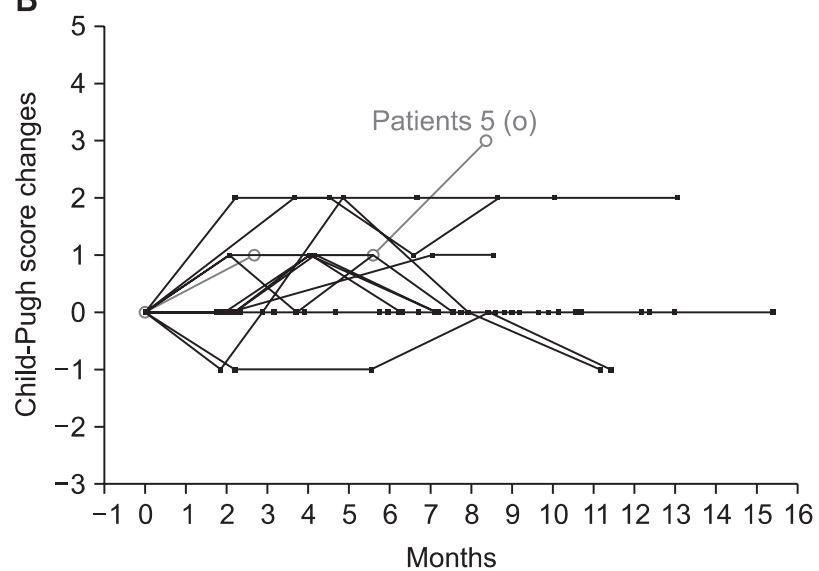

Fig. 3. The absolute (A) and relative (B) the change of Child-Pugh score along the follow-up periods.

in patients with HCC ineligible for standard therapies such as resection, transplantation and RFA. However, as in patient \#5 with baseline CP score of 5 , though it is not sure that the progression of $L C$ and the marked shrinkage of liver volume is related with $R T$ in the patient, the application of RT as a curative treatment has to be decided carefully only for patients with sufficient clinical liver function reserve and sufficiently less normal liver irradiation achievable.

While applying the RT as a palliative treatment for advanced $\mathrm{HCC}$, it is not infrequent to observe the shrinkage of HCC itself and the adjacent normal liver included in the treated volume, accompanied by compensatory hypertrophy of the nontumor liver $[16,17]$. The temporal balance after RT between the shrinkage of adjacent normal liver and the hypertrophy of non-tumor liver is important in the preservation of clinical liver function as compensated. Sometimes the compensatory hypertrophy after RT provides a beneficial condition for surgical resection for advanced HCC [16]. The factors associated with liver regeneration such as the extent of resection have been evaluated in the surgical settings [18]. However, the impact of RT related factors associated with the temporal balance between ipsilateral shrinkage and contralateral hypertrophy of normal liver after RT has seldom been addressed [18]. At least, the partial liver irradiation with sufficiently small treated volume as in the current study did not result the considerable shrinkage of ipsilateral normal liver and the contralateral compensatory hypertrophy. Further studies on the RT related factors associated with liver volume change are warranted.

RILD is one of the most important treatment-related complications in patients receiving RT for HCC [19]. The dose volumetric guidelines to prevent RILD were reported by several investigators $[11,20]$. Recently, with the help of these guidelines and high precision radiation delivery in the treatment of HCC, the classical RILD is reported less frequently. The CP score is a well-known prognostic factor in patients with $\mathrm{LC}$. And CP score is used as a measure of clinical liver function, thus we hypothesized that $\mathrm{CP}$ score changes could reflect the change of clinical liver function after RT especially in patients who have shown non-classical RT induced liver damage. In the current study, the patient died of LC progression showed the increase of CP score more than 3 after RT for small HCC. It is believed that the CP score change can be used for FU of liver function along with the change of liver volume.

Recently, there were discussions on the paradigm shift in RT role for HCC from palliative to curative by several investigators. Dawson [14] pointed out that RT could be used not only for palliative treatments but for potentially curative options in small to medium sized HCC. The highest chance of tumor control after RT is paradoxically in patients with small $\mathrm{HCC}<3 \mathrm{~cm}$; the tumoricidal doses of RT are possible to deliver safely [14]. To the traditional palliative application, Dawson [14] added the items of bridging RT prior to transplantation; salvage RT for HCC refractory to other therapies and definitive RT for HCC unsuitable for standard curative therapies, as the RT role in the treatment of HCC. For these ambitions to become a clinical reality, RT should be decided only for patients in whom RT could be delivered safely enough besides the tumoricidal radiation doses.

With the application of 4-dimensional conformal RT techniques considering patient's breathing, the high dose hypofractionated RT such as SBRT exert the potential role for curative RT in patients with small HCC [13]. Furthermore, the role of particle therapy is expected promising in the near 
future. Particle therapy is another example of a technologic advance that should benefit HCC patients by reducing the integral radiation dose to the liver, facilitating treatment of larger tumors, and allowing more patients to be treated safely with high doses [21]. Further volumetric studies using particle therapy such as proton therapy is warranted to investigate that the particle therapy is safe and effective modality in the treatment of small HCC.

Limitations of the current study were the small number of patients and retrospective nature. In the mixed model analysis, the temporal change of the liver volume was not statistically significant. It could be due to that the liver volume was not affected by high dose hypofractionated RT for small HCC or the number of study population was not sufficiently large to reveal the temporal volume change. And there are possibilities that the liver volumes were affected not only by RT but also intra-hepatic recurrences followed by salvage treatments such as TACE. However, in our series, mean recurrent tumor diameter was $1.40 \mathrm{~cm}$ in in-field recurrences and $1.93 \mathrm{~cm}$ in intra-hepatic elsewhere recurrences. So, the average extent of liver volume change due to intrahepatic recurrences are around $30 \mathrm{~cm}^{3}$. It is believed that the intra-hepatic recurrences didn't influence enough to make the considerable change of entire liver volume.

In conclusion, the changes of liver volume and clinical liver function in the form of CP score after high dose hypofractionated RT in patients with small HCC was not profound in this study population. The high dose hypofractionated RT for small HCC is relatively safe and feasible in terms of liver volumetric changes and clinical liver function.

\section{Conflict of Interest}

No potential conflict of interest relevant to this article was reported.

\section{Acknowledgments}

This research was supported by Basic Science Research Program through the National Research Foundation of Korea (NRF) funded by the Ministry of Education, Science and Technology (2011-0004444).

\section{References}

1. Korean Liver Cancer Study Group and National Cancer Center,
Korea. Practice guidelines for management of hepatocellular carcinoma 2009. Korean J Hepatol 2009;15:391-423.

2. Kokudo N, Makuuchi M. Evidence-based clinical practice guidelines for hepatocellular carcinoma in Japan: the J-HCC guidelines. J Gastroenterol 2009;44 Suppl 19:119-21.

3. Forner $A$, Reig $M E$, de Lope $C R$, Bruix J. Current strategy for staging and treatment: the BCLC update and future prospects. Semin Liver Dis 2010;30:61-74.

4. Zhou XD, Tang ZY, Yang BH, et al. Experience of 1000 patients who underwent hepatectomy for small hepatocellular carcinoma. Cancer 2001;91:1479-86.

5. Llovet JM, Fuster J, Bruix J. Intention-to-treat analysis of surgical treatment for early hepatocellular carcinoma: resection versus transplantation. Hepatology 1999;30:1434-40.

6. Kudo M. Radiofrequency ablation for hepatocellular carcinoma: updated review in 2010. Oncology 2010;78 Suppl 1:11324.

7. Park HC, Seong J, Han KH, Chon CY, Moon YM, Suh CO. Doseresponse relationship in local radiotherapy for hepatocellular carcinoma. Int J Radiat Oncol Biol Phys 2002;54:150-5.

8. Kim DY, Park W, Lim DH, et al. Three-dimensional conformal radiotherapy for portal vein thrombosis of hepatocellular carcinoma. Cancer 2005;103:2419-26.

9. Park W, Lim DH, Paik SW, et al. Local radiotherapy for patients with unresectable hepatocellular carcinoma. Int J Radiat Oncol Biol Phys 2005;61:1143-50.

10. Mornex F, Girard N, Beziat C, et al. Feasibility and efficacy of high-dose three-dimensional-conformal radiotherapy in cirrhotic patients with small-size hepatocellular carcinoma non-eligible for curative therapies: mature results of the French Phase II RTF-1 trial. Int J Radiat Oncol Biol Phys 2006; 66:1152-8.

11. Kim TH, Kim DY, Park JW, et al. Dose-volumetric parameters predicting radiation-induced hepatic toxicity in unresectable hepatocellular carcinoma patients treated with threedimensional conformal radiotherapy. Int J Radiat Oncol Biol Phys 2007;67:225-31.

12. Bae SH, Park HC, Lim DH, et al. Hypofractionated radiotherapy for small-sized hepatocellular carcinoma as salvage therapy: sustained local control and safety. J Korean Soc Ther Radiol Oncol 2010;28:85-90.

13. Bae SH, Park HC, Lim do H, et al. Salvage treatment with hypofractionated radiotherapy in patients with recurrent small hepatocellular carcinoma. Int J Radiat Oncol Biol Phys 2012;82:e603-7.

14. Dawson LA. Overview: where does radiation therapy fit in the spectrum of liver cancer local-regional therapies? Semin Radiat Oncol 2011;21:241-6.

15. Lee IJ, Seong J. Radiotherapeutic strategies in the management of hepatocellular carcinoma. Oncology 2011;81 Supp 
1:123-33.

16. Seong J, Park HC, Han KH, et al. Clinical results of 3-dimensional conformal radiotherapy combined with transarterial chemoembolization for hepatocellular carcinoma in the cirrhotic patients. Hepatol Res 2003;27:30-5.

17. Gaba RC, Lewandowski RJ, Kulik LM, et al. Radiation lobectomy: preliminary findings of hepatic volumetric response to lobar yttrium-90 radioembolization. Ann Surg Oncol 2009;16: 1587-96.

18. Choi JH, Kim K, Chie EK, et al. Does adjuvant radiotherapy suppress liver regeneration after partial hepatectomy? Int J Radiat Oncol Biol Phys 2009;74:67-72.
19. Lawrence TS, Robertson JM, Anscher MS, Jirtle RL, Ensminger WD, Fajardo LF. Hepatic toxicity resulting from cancer treatment. Int J Radiat Oncol Biol Phys 1995;31:1237-48.

20. Liang $S X$, Huang $X B, Z$ Zhu $X D$, et al. Dosimetric predictor identification for radiation-induced liver disease after hypofractionated conformal radiotherapy for primary liver carcinoma patients with Child-Pugh Grade A cirrhosis. Radiother Oncol 2011;98:265-9.

21. Dawson LA. Protons or photons for hepatocellular carcinoma? Let's move forward together. Int J Radiat Oncol Biol Phys 2009;74:661-3. 